\title{
RISCOS OCUPACIONAIS E OS RESÍDUOS DE SERVIÇOS DE SAÚDE EM CENTRO CIRÚRGICO
}

\author{
Occupational risks and health care waste in the surgery center
}

\author{
Riesgos ocupacionales y residuos de salud en un centro quirúrgico
}

\author{
Graciele Pinheiro de Morais Justiniano ${ }^{1}$ (D), Aline Helena Appoloni Eduardo 2 (D), Cibele Correia Semeão Binotto ${ }^{3}$ (D), \\ Juice Ishie Macedo ${ }^{4}$ (D), Tatiane Bonametti Veiga ${ }^{5}$ (D), Silvia Helena Tognolib ${ }^{\text {ID }}$, Adriana Aparecida Mendes ${ }^{7 *}$
}

RESUMO: Objetivo: Conhecer a relação dos riscos ocupacionais com os resíduos de serviços de saúde (RSS), na perspectiva de profissionais de enfermagem que atuam no centro cirúrgico (CC). Método: Trata-se de uma pesquisa de campo de caráter exploratório e descritivo e abordagem qualitativa. Os dados foram coletados por meio de entrevista gravada, com três questões abertas sobre a geração e o manejo de resíduos e riscos à saúde dos profissionais de enfermagem. A amostra foi composta de 11 técnicos e dois auxiliares de enfermagem. Os dados foram organizados e analisados segundo a proposta do método do discurso do sujeito coletivo (DSC). Resultados: Os discursos revelaram preocupação em realizar o manejo adequado dos resíduos gerados, com destaque para os biológicos e perfurocortantes. Os profissionais compreendem que o não cumprimento de práticas seguras de manejo pode expor prejuízos à própria saúde, à do paciente e à de outros profissionais. Conclusão: Os resultados evidenciaram exposição dos profissionais de enfermagem aos resíduos gerados no CC, o que reforça a necessidade de práticas contínuas de orientações e treinamentos para o manejo apropriado desses resíduos, com a finalidade de minimizar riscos de exposição dos profissionais, dos pacientes e do ambiente.

Palavras-chave: Saúde do trabalhador. Riscos ocupacionais. Resíduos de serviços de saúde. Centros cirúrgicos. Enfermagem.

ABSTRACT: Objective: To understand the relationship between occupational risks and health care waste (HCW), from the perspective of nursing professionals working in the surgery center (SC). Method: This is exploratory and descriptive field research with a qualitative approach. Data were collected through recorded interviews, with three open questions on the generation and management of waste and health risks to nursing professionals. The sample consisted of 11 technicians and two auxiliary nurses. Data were organized and analyzed according to the collective subject discourse (CSD) method. Results: The speeches revealed concern in adequately managing the generated waste, particularly biological and sharp ones. Professionals understand that failure to comply with safe management practices can lead to damage to their health, that of the patient, and that of other professionals. Conclusion: The results showed the exposure of nursing professionals to the waste generated in the SC, which reinforces the need for ongoing guidance practices and training for the appropriate management of these residues, in order to minimize risks of exposure for professionals, patients, and the environment. Keywords: Occupational health. Occupational risks. Medical waste. Surgicenters. Nursing.

RESUMEN: Objetivo: Conocer la relación entre riesgos laborales y los residuoe de los servicios de salud (RSS), desde la perspectiva de los profesionales de enfermería que trabajan en quirófano (CQ). Método: Esta es una investigación de campo exploratoria y descriptiva con un enfoque cualitativo. Los datos se recopilaron mediante entrevistas grabadas, con tres preguntas abiertas sobre la generación y gestión de residuos y riesgos para la salud de los profesionales de enfermería. La muestra consistió en 11 técnicos y dos auxiliares de enfermería. Los datos fueron organizados y analizados de acuerdo con el método del discurso del sujeto colectivo (DSC). Resultados: Los discursos revelaron una preocupación por llevar a cabo el manejo adecuado de los

'Enfermeira pela Universidade de Araraquara (UNIARA) - Araraquara (SP), Brasil.

2Enfermeira, doutora em Ciência pela Escola de Enfermagem de Ribeirão Preto da Universidade de São Paulo. Docente do curso de Enfermagem da Universidade Federal de São Carlos (UFSCar) - São Carlos (SP), Brasil. ${ }^{3}$ Enfermeira, doutoranda pela Universidade Federal de São Carlos. Docente do curso de Enfermagem da UNIARA - Araraquara (SP), Brasil.

${ }^{4}$ Enfermeira, doutora em Enfermagem pela Universidade Federal de Minas Gerais. Docente aposentada da Universidade Estadual do Centro-Oeste - Guarapuava (PR), Brasil.

${ }^{5}$ Engenheira civil, doutora em Ciência pela Escola de Enfermagem de Ribeirão Preto da Universidade de São Paulo (USP). Docente da Universidade Estadual do Centro-Oeste - Irati (PR), Brasil.

${ }^{\circ}$ Enfermeira, doutoranda da Escola de Enfermagem de Ribeirão Preto da USP - Ribeirão Preto (SP), Brasil.

${ }^{7}$ Enfermeira, doutora em Ciência pela Escola de Enfermagem de Ribeirão Preto da USP. Docente do curso de Enfermagem da UNIARA - Araraquara (SP), Brasil.

*Autora correspondente: adrianaapmendes@yahoo.com.br

Recebido: 13/08/2019 - Aprovado: 29/01/2020

DOI: $10.5327 / Z 1414-4425202000010005$ 
desechos generados, con énfasis en productos biológicos y objetos punzantes. Los profesionales entienden que el incumplimiento de las prácticas de gestión segura puede exponer el daño a su propia salud, la del paciente y la de otros profesionales. Conclusión: Los resultados evidenciaron la exposición de los profesionales de enfermería a los desechos generados en el CQ, lo que refuerza la necesidad de orientación continua y prácticas de capacitación para el manejo adecuado de estos desechos, a fin de minimizar los riesgos de exposición de profesionales, pacientes y medio ambiente Palabras clave: Salud laboral. Riesgos laborales. Residuos sanitarios. Centros quirúrgicos. Enfermería.

\section{INTRODUÇÃo}

O cuidado com a saúde deve ser um ato seguro, minimizando a ocorrência de eventos adversos. Entretanto, no cotidiano laboral, há exposição excessiva a situações que oferecem risco à saúde do profissional, com destaque para aquelas ocorridas no centro cirúrgico (CC), local em que o trabalhador está vulnerável a ocorrências danosas ${ }^{1}$.

Pontua-se que os profissionais de enfermagem representam número expressivo dos trabalhadores no CC e estão expostos a diversas situações que são geradoras de desgastes. Ressalta-se que fatores de risco presentes nesse tipo de ambiente podem ocasionar prejuízos à qualidade da produção e da assistência prestada nas diferentes situações, comprometendo diretamente a saúde dos trabalhadores. Esses fatores de risco resultam da exposição e do manuseio de agentes físicos, químicos, biológicos, ergonômicos e psicossociais, ocasionando acidentes ocupacionais ${ }^{2-5}$.

Diante dessa realidade, vale destacar os riscos relacionados à exposição aos resíduos de serviços de saúde (RSS), gerados continuamente no CC, decorrentes de diferentes tipos de procedimentos realizados durante a prática assistencial.

De acordo com a Resolução da Diretoria Colegiada (RDC) nº 222/2018, da Agência Nacional de Vigilância Sanitária (ANVISA), geradores de RSS englobam todos os serviços relacionados com o atendimento à saúde humana e animal, seja na assistência domiciliar, seja em laboratórios analíticos de produtos para saúde, necrotérios, funerárias, unidades móveis de atendimento, entre outros similares ${ }^{6}$.

Segundo o que determina a RDC $\mathrm{n}^{\circ} 222 / 2018$, os RSS são classificados em diferentes grupos:

- grupo A: resíduos biológicos, que agregam substâncias infectantes em sua composição;

- grupo B: resíduos químicos, tais como medicamentos e saneantes;

- grupo C: resíduos radioativos, representados pelos produtos que possam conter radionuclídeos em valores superiores aos estabelecidos pela Comissão Nacional de Energia Nuclear;

- grupo D: resíduos comuns, que não apresentam nenhuma das características dos outros grupos;
- grupo E: resíduos perfurocortantes, tais como lâminas, agulhas, entre outros ${ }^{6}$.

Pontua-se que no CC são gerados todos os tipos de resíduos. Entre os profissionais, aqueles que compõem a equipe de enfermagem estão em contato diariamente com os resíduos ao realizar procedimentos em sua prática, expondo-os aos riscos relacionados a tais materiais ${ }^{7}$. Destaca-se que a equipe de enfermagem está representada em maior número e é responsável pelo direcionamento do fluxo dos resíduos ${ }^{8}$.

Diante dessa realidade, o gerenciamento dos RSS constitui-se em um conjunto de procedimentos de gestão devidamente planejados e implementados com referência em bases científicas, técnicas, normativas e legais cujas finalidades são diminuir a geração de resíduos e proporcionar a disposição final adequada a eles, em benefício da segurança, da saúde dos profissionais, dos pacientes e do ambiente ${ }^{6}$.

Convém ressaltar que o número de estudos que investigam a problemática dos RSS entre os profissionais de enfermagem em CC ainda é reduzido, sendo necessário explorar esse tipo de pesquisa como forma de fortalecer a prática do manejo dos resíduos gerados, minimizando a exposição de profissionais, pacientes e ambiente a eles.

\section{OBJETIVO}

Conhecer a relação dos riscos ocupacionais com os RSS na perspectiva de profissionais de enfermagem que atuam no CC.

\section{MÉTODO}

Trata-se de uma pesquisa de campo de caráter exploratório e descritivo e com abordagem qualitativa realizada em junho de 2016, em um CC de um hospital no interior de São Paulo.

Foram convidados a participar do estudo os 32 profissionais de enfermagem (enfermeiros, técnicos e auxiliares de enfermagem) atuantes no CC selecionado, os quais foram abordados em horário e local reservados, conforme 
previamente acordado com a coordenação do setor, sem interferir na rotina laboral.

Os critérios de inclusão foram: atuar no CC por tempo superior a 12 meses, aceitar participar do estudo e estar na unidade durante o período de coleta dos dados. Quanto à exclusão, aqueles que estavam em férias, folga ou fora da unidade de trabalho por algum motivo, no período da coleta dos dados, não puderam fazer parte da pesquisa.

Entre os 32 profissionais, 19 não atenderam aos critérios de inclusão, chegando-se ao total de 13 profissionais participantes, sendo 11 técnicos e dois auxiliares de enfermagem.

Para a coleta de dados, elaborou-se um instrumento com três questões abertas, segundo a proposta do método do discurso do sujeito coletivo (DSC) ${ }^{9}$ :

1. Nas atividades realizadas no CC, são realizados diferentes tipos de procedimentos não invasivos e invasivos que geram resíduos. Conte-me sobre esses resíduos;

2. Em seu cotidiano laboral no CC, você realiza procedimentos não invasivos e invasivos que geram resíduos, sendo necessário o manejo (manuseio) adequado para que não ocorra nenhum tipo de prejuízo à sua saúde, do paciente e do ambiente. Conte-me como você realiza esse manejo (manuseio);

3. No ambiente do CC, os procedimentos que são realizados geram resíduos que podem oferecer riscos à sua saúde. Você poderia falar sobre esses riscos?

A coleta de dados individual ocorreu após aprovação do Comitê de Ética em Pesquisa, segundo as recomendações da Resolução $n^{\circ}$ 466/2012 ${ }^{10}$, com parecer 1.447.657 e Certificado de Apresentação para Apreciação Ética(CAAE) 53337316.0.0000.5383. Após assinatura do Termo de Consentimento Livre e Esclarecido, as informações foram coletadas nos períodos da manhã, tarde e noite e registradas em gravador de voz, com tempo médio de três minutos e dez segundos para cada entrevista.

Para a análise dos dados, utilizou-se o método do DSC, sendo as entrevistas gravadas e transcritas manualmente. Em seguida, extraíram-se as expressões-chave, que representam os principais trechos ou segmentos encontrados nas transcrições integrais das respostas obtidas no conteúdo principal, e selecionaram-se as ideias centrais, com posterior construção dos discursos .

\section{RESULTADOS}

Perante os resultados obtidos nas entrevistas, as três questões norteadoras deram origem a seis DSC, apresentados a seguir.

\section{DSC 1: Resíduos gerados e exposição dos profissionais a riscos ocupacionais}

Os profissionais de enfermagem apontaram os diferentes tipos de resíduos gerados no CC e a preocupação com situações de risco de exposição.

Perfuro, outros tipos [...], os restos das amputações pequenas, peles [...], anatomopatológicos, produtos químicos, medicamentos, os descartáveis [...], também [...] agulhas [...], Abocaths ${ }^{\circledR}[\ldots]$, acessos centrais que o doutor passa [...] que, às vezes, geram um risco [...] para o funcionário [...], médicos, não sendo bem manipulados [...], na correria deixam expostos [...]. Todos os procedimentos geram bastante residuos [...]. Em uma cirurgia de fratura de fêmur [...] são usadas muitas compressas para estancar o sangramento e [...] são jogadas no hamper [...]. Elas são sempre bem sujas [...], causam muita sujeira na sala e têm muito resíduo [...]. Também é usado bastante fio, como na cirurgia cardíaca [...], em que se abrem muitas caixas $[\ldots]$, pinças [...] e vários materiais diferentes [...], luva, gaze [...], campo, agulha, seringa, dreno [...]. Tem os residuos comuns [...], que são papel toalha, que a gente usa durante o procedimento de lavagem das mãos [...]. O resíduo que é o lixo estéril, porque aqui nós utilizamos campo estéril descartável, avental [...]. Então, isso gera um resíduo [...]. Tem o sólido, que são os perfurocortantes [...], os contaminados [...], comuns [...], ampolas de vidro [...], vários tipos de resíduos [...]. Tudo que tem sujidade com sangue, contato com paciente [...], secreção [...], as lâminas.

\section{DSC 2: Exposição dos profissionais a acidentes ocupacionais com resíduos perfurocortantes}

Os participantes do estudo revelaram que, no cotidiano laboral, entre os resíduos gerados, os perfurocortantes podem levar a riscos ocupacionais, comprometendo sua saúde. 
se perfurar, eu mesmo já me perfurei $[\ldots]$ por não ter jogado em lugar adequado, não [...] organizarem na sua mesa [...]. A gente tem que tomar muito cuidado para não deixar o Descarpack ${ }^{\circledR}$ lotado, porque a gente pode se machucar [...], machucar o colega que leva isso [...]. Os resíduos perfurocortantes [...] devem ser descartados no Descarpack ${ }^{\circledR}[\ldots]$, os contaminados, [...] jogados no lixo branco $[\ldots]$, os $[\ldots]$ comuns $[\ldots]$ no lixo preto.

\section{DSC 3: Cuidado dos profissionais de enfermagem ao manusear os resíduos gerados}

Outra preocupação dos participantes identificada nas respostas foi com a necessidade do manuseio seguro dos diferentes tipos de materiais, com a finalidade de minimizar a exposição a riscos.

Quando a gente manuseia [...], tenta tomar possiveis cuidados [...]. É um pouco dificil, porque o CC é um setor que [...] não para [...]. Então, nem sempre vê um perfuro na mesa, e onde a gente acaba perfurando [...], se machucando [...]. A gente tenta prevalecer a nossa saúde e a do paciente, não mexer nesses lixos quando o paciente está em sala, procura mandar o paciente para a sala de recuperação, depois fazer a retirada de todos esses materiais [...], prestar mais atenção na mesa ao retirar [...], olha tudo novamente, se já colocou na caixa para descer para central. [...] Todo manuseio com esse tipo de resíduo a gente presta muita atenção, sempre com luva. Dependendo de como é o residuo e a quantidade, eu costumo colocar duas luvas, para não ter o problema de furar uma e sujar minha mão [...]. O manuseio é o descarte no lugar correto, [...] mas quando está na mesa de cirurgia sempre pego com uma pinça. O bisturi a gente sempre tira com um porta-agulha para não ter risco de pular e machucar alguém ou você mesma [...], não acontecer um acidente [...]. Nunca pego com a mão, porque mesmo que você perfurar o dedo com luva [...] teu dedo está lá, porque a luva só não protege nada basicamente [...], como utiliza muitos campos. A gente tem que tomar o máximo de cuidado possível [...], tem que utilizar luva [...] de procedimento [...]. Se for uma seringa com agulha, não pode fazer o reencape, você tem que jogar a seringa com tudo [...], sempre com luva e, dependendo, com máscara também, para evitar respingos $[\ldots]$ na gente.

\section{DSC 4: Riscos ocupacionais relacionados ao descarte dos resíduos}

Os participantes revelaram preocupação com a necessidade de atenção no momento do descarte dos resíduos e alertaram sobre ela, pois compreendem que há riscos nessa prática.

"Ás vezes" o residente ou interno fala que já tirou o perfuro. A gente vai ver [e] não tirou. [...] Na hora de retirar os lixos, até mesmo [...] o reciclável, porque às vezes alguém pode passar e jogar alguma coisa no reciclável [...], a gente acaba se colocando em risco [...]. Olha o chão, observa..., porque tem alguns que deixam cair no chão [...]. Quando a gente encontra, a gente pega, lógico, calça a luva, tem que ter cuidado, pega de um jeito para não correr riscos e descarta no lugar adequado [...]. Tem pote de secreção. Às vezes na cirurgia o doutor já vai aspirando no pote [...] para gente depois já desprezar, o descarte da bolsa de diurese [...], o dreno de Portovac ${ }^{\circledR}$ [...] no vaso sanitário, que é do expurgo, [...] tomando muito cuidado [...], com luva e, geralmente dependendo do que é, como uma lâmina em bisturi, com uma pinça Kelly [...], desprezo [...] no Descarpack ${ }^{\circledR}[\ldots]$, e demais a gente coloca no devido saco transparente os recicláveis e no branco os contaminados [...], as roupas no saco azul. [...] É um procedimento aqui do hospital, e a gente tem a separação do lixo reciclável, que são as embalagens e tudo mais que a gente separa no saco transparente.

\section{DSC 5: Insegurança dos profissionais de enfermagem relacionada à ausência de informações sobre o paciente}

A carência de informações sobre as condições clínicas do paciente atendido no CC foi mencionada pelos profissionais, reforçando a necessidade da adesão aos equipamentos de proteção individual. Sempre colocando luva, máscara, avental, que a gente não
sabe o que o paciente tem. Às vezes o paciente chega de
urgência e sobe direto [...], não sabemos as doenças que
esse paciente tem. Aqui é como uma porta de entrada,
como se fosse uma emergência [...]. Sabemos que há os
riscos biológicos. Na maioria das vezes, quando o paciente
tem tuberculose, nós não sabemos, não usamos máscara, 
porque não temos esse protocolo para todos os pacientes [...]. Quando você sabe, já foi diagnosticado, já passou por aqui, já tá no quarto [...], mas muitas das vezes você lida com paciente sem saber [...]. A gente pode se contaminar com secreções que às vezes ficam, pode espirrar no olho, trazendo pra gente alguma doença [...]. Então, você se protege, põe luva, usa máscara [...]. Por mais que você use os paramentos certos, [...] corre risco mesmo assim de contrair alguma coisa do paciente sem saber.

\section{DSC 6: Riscos relacionados aos resíduos gerados}

A necessidade de manter práticas de manuseio adequado dos resíduos durante as atividades assistenciais foi apontada pelos profissionais.

Como falei, a gente tenta não fornecer riscos nem para gente [...], o paciente [...], ninguém que está na sala [...]. A gente corre o risco o tempo inteiro [...]. Preocupação daqui do ambiente de trabalho e que a [Comissão de Controle de Infecção Hospitalar] CCIH preza muito pra gente sempre estar usando óculos, máscara, por conta desse risco de contaminação [...], de pegar algum resíduo no olho, $[. .$.$] ou de secreção [...]. A gente tem esses agora$ com dispositivos, que são mais práticos, você recolhe agulha, às vezes pode esquecer, é um risco? É! Mas, assim, de se perfurar é bem menos [...]. É uma segurança maior para nós também de acidente [...]. Antigamente, quando eles não eram reencapáveis, acontecia isso de se perfurar [...]. O lixo também não descartado no lugar certo [...], não recolhido nos horários, lógico que vai acumulando, isso pode sim gerar riscos [...], ter um perfurocortante $e[\ldots]$ pode se perfurar. E eu acredito que seria mais do perfurocortante em si que os materiais. Se você usar os [equipamentos de proteção individual] EPIs, não tem perigo de espirrar sangue ou alguma coisa do tipo [...]. Risco [...] de tudo, doença [...], o principal, hepatite [...]. Às vezes tem procedimento cirúrgico que é cirurgia contaminada [...], essas Pseudomonas [...] são as que mais têm risco pra gente [...], tipos de bactérias fortes que tem nos hospitais, hepatite $C$, [vírus da imunodeficiência adquirida] HIV, tudo, sifilis também [...]. Riscos biológicos, que são dos resíduos sólidos [...], agulhas, fios de sutura, que a gente pode se perfurar [...], se cortar e se contaminar [...], e também outros tipos de acidente. Conforme a gente vai desprezar uma diurese
[...], colocando os campos dentro do hamper, pode voar secreção, é um risco muito grande [...]. Há vários riscos, porque os fluidos [...], todos esses [...], tem que tomar muito cuidado [...]. Você pode estar se contaminando, exceto os recicláveis, desde que eles não estejam com sujidade, ou seria contaminado [...] se não tiver protegido, os inalatórios, dependendo no que você está mexendo [...]. Para os pacientes, os riscos gerados, eu acho, uma agulha mal descartada. Em relação a [...] aerossóis, são [...] essas doenças transmitidas pelo ar [...], o produto químico de cair na pele dá uma alergia [...]. Separar o lixo direito, porque uma luva que a gente coloca no saco que deveria ser descartável acaba contaminando tudo. Então, a hora que chega para o pessoal lá de baixo [...] eles veem uma luva ou uma gaze dentro do material reciclável, acaba indo para o material contaminado [...], acaba pagando mais para [...] o descarte certo no lixo.

\section{DISCUSSÃO}

Neste estudo, a preocupação com a exposição aos resíduos gerados no CC pelos profissionais de enfermagem foi evidenciada nos discursos, assim como a atenção para atender às normas implementadas pela instituição para a não exposição aos riscos ocupacionais.

O estudo da percepção dos riscos inerentes aos resíduos gerados nos estabelecimentos de assistência à saúde é relevante para a elaboração e a implementação de ações que minimizem danos aos trabalhadores, à comunidade e ao ambiente ${ }^{11}$.

Outra situação identificada no discurso dos participantes relacionava-se à geração de resíduos continuamente por 24 horas, pois se trata de uma unidade de atendimento a pacientes em situações de urgência e emergência, sendo esses resíduos gerados em diferentes momentos das práticas assistenciais $\mathrm{e}$ dos procedimentos realizados pela equipe multiprofissional.

Os participantes ainda citaram que, entre a diversidade dos resíduos gerados, há também os gases anestésicos, bem como a possibilidade de exposição do profissional a radiações, situações de risco que podem interferir no desempenho profissional e na qualidade de vida do trabalhador.

Segundo dados de estudo realizado em um hospital público e universitário, a exposição dos trabalhadores de enfermagem a acidentes ocupacionais pode ocorrer nas atividades cotidianas em resposta à carga de trabalho. Essa exposição está associada aos fatores químicos, físicos, psíquicos e mecânicos, 
especialmente os biológicos, que também podem ocasionar doenças ocupacionais ${ }^{12}$.

Ainda quanto à possibilidade de exposição ocupacional dos profissionais de enfermagem atuantes no CC aos diferentes tipos de resíduos gerados durante as práticas assistenciais, os participantes afirmaram que o risco aumenta em situações em que não há manejo adequado desses materiais.

Entre os resíduos gerados, destacaram-se os perfurocortantes, pois são aqueles que os profissionais da equipe de enfermagem manuseiam constantemente e que podem oferecer riscos à sua saúde, despertando preocupação nessa categoria profissional.

O manejo inadequado dos resíduos gerados nos estabelecimentos de saúde, particularmente em alguns setores, como banco de sangue, salas de cirurgia e laboratórios, representa riscos para as pessoas e para o ambiente em razão das suas características, pois podem conter agentes infectantes, radioativos, perfurocortantes, entre outros ${ }^{13}$.

Outro estudo realizado em Unidades de Saúde da Família no município de São Carlos, interior de São Paulo, aponta que a exposição a materiais potencialmente contaminados, entre eles os perfurocortantes, é frequente nas atividades do profissional de enfermagem, fato relacionado à excessiva manipulação desse tipo de material no cotidiano laboral ${ }^{14}$.

Assim, conforme descrito na Norma Regulamentadora (NR) $32 / 2005$, que determina normas para a segurança e a saúde dos trabalhadores nos estabelecimentos de assistência à saúde no que se refere à exposição aos materiais perfurocortantes, um plano de ação direcionado para minimizar esse tipo de contato deve ser implementado e supervisionado ${ }^{15}$.

Também, dados de estudo realizado em hospital universitário em João Pessoa, na Paraíba, com a finalidade de identificar os principais tipos de acidentes que acometem os profissionais de enfermagem, revelaram que é prevalente a exposição aos perfurocortantes, seguida do contato com secreções de pacientes, agressões, entre outros. Trata-se de situações que interferem na qualidade de saúde dos profissionais e, em alguns casos, resultam em afastamento ${ }^{16}$.

Estudo feito em um hospital público no interior do Ceará identificou que, entre os resíduos gerados, as etapas de manejo, constituídas de segregação, acondicionamento e identificação para os perfurocortantes, são seguidas corretamente. De acordo com os autores, no que se refere à exposição ocupacional a esse tipo de resíduo, é possível afirmar que há preocupação dos profissionais envolvidos ${ }^{17}$.

Quanto ao cuidado em manusear os resíduos, o discurso dos participantes desta pesquisa revelou que há atenção e esta está direcionada para tal prática em momentos distintos, desde a geração até o descarte em recipiente adequado, como forma de proteção à saúde nas situações de atendimento.

Estudo realizado na Universidade Federal de Minas Gerais com graduandos do Departamento de Ciências Biológicas e da Saúde, com a finalidade de identificar o conhecimento desses alunos no que se refere ao gerenciamento dos resíduos dos grupos A biológicos e E perfurocortantes, revelou que o conhecimento parcial sobre o descarte adequado dos perfurocortantes desperta preocupação, pois os participantes da pesquisa representam um grupo responsável por exposição ocupacional ${ }^{11}$.

No tocante ao cumprimento das etapas de manejo, identificaram-se, no discurso dos participantes, preocupação e apreensão quanto à sua segurança, pois eles têm consciência de que estão expostos a diferentes tipos de riscos, entre eles aos resíduos gerados. Estão cientes também de que as etapas de manejo devem ser conduzidas com segurança, com o objetivo de preservar sua saúde, a do paciente e a de outros profissionais que atuam no CC. Ressalta-se que as etapas de manejo estão contempladas no gerenciamento desses resíduos ${ }^{6}$.

Outro estudo, realizado em hemocentro no Paraná, com o propósito de conhecer o gerenciamento dos RSS gerados no serviço, evidenciou a necessidade de um olhar diferenciado para as etapas de manejo, assim como para o fortalecimento da prática de educação permanente em serviço, pois há riscos para a saúde pública e para o ambiente ${ }^{18}$.

Neste estudo, no que tange à prática, outra preocupação revelada foi quanto ao cumprimento do descarte adequado dos resíduos, pois, quando o descarte correto não ocorre e o resíduo comum é descartado como contaminado, há aumento do custo para a disposição final adequada.

Assim, é possível afirmar que, quando a segregação não ocorre de forma adequada, as outras etapas de manejo são comprometidas, havendo risco de exposição para o paciente, o profissional e o ambiente, se os resíduos forem descartados sem tratamento prévio ou em local inadequado ${ }^{17,19,20}$.

Todavia, o profissional de enfermagem não é o único responsável pela geração de resíduos no momento da assistência prestada ao paciente; profissionais de outras áreas da saúde também os geram. À enfermagem, porém, são delegadas as responsabilidades de manusear e acondicionar esses resíduos, pois é ela a encarregada pela continuidade da assistência durante o processo de tratamento e recuperação do paciente.

Considerando a prática do manejo dos resíduos gerados pelos profissionais de enfermagem, é possível afirmar que eles sejam o ponto de partida na gestão dos RSS, visto que a segregação, etapa inicial do manejo, é, em sua maioria, realizada por esses profissionais`. 
Nessa direção, pontua-se a necessidade de conhecimento do profissional de enfermagem sobre as etapas de manejo contidas no gerenciamento dos resíduos, como descreve a $\mathrm{RDC} \mathrm{n}^{\circ}$ $222 / 2018^{6}$, minimizando a possibilidade de exposição ocupacional.

Outro conteúdo indicado no presente estudo foi a questão da insegurança identificada no discurso dos participantes em relação ao paciente assistido. Conforme relatos, os profissionais permanecem em situação de alerta no bloco cirúrgico, pois a admissão do paciente nesse ambiente pode ocorrer a qualquer momento, seja em situações de urgência, seja em emergência, em diferentes níveis de complexidade das condições de saúde. Esse fato, segundo os profissionais da área, não deixa margem de tempo hábil para comunicação prévia ao período que antecede as intervenções no CC, o que torna essa situação propensa à exposição a riscos.

Perante essa exposição, os profissionais citaram que, para sua própria segurança, colocam em prática ações de prevenção, como o uso de EPI de forma correta, como meio para minimizar a exposição a esse tipo de ocorrência adversa e, também, recebem continuamente atenção e cuidado da CCIH.

Os resíduos produzidos no CC são resultantes de procedimentos realizados durante os procedimentos anestésico-cirúrgicos. São exemplos os aventais descartáveis, os campos cirúrgicos, as compressas, as gazes, os invólucros dos materiais utilizados, entre outros ${ }^{21}$.

Assim, a realização de procedimentos de maneira mecanizada, acelerada ou distraída pode levar o profissional a não considerar sua segurança e sua saúde no ambiente de trabalho. No CC, a necessidade da prática das ações assistenciais de forma intensa predispõe os profissionais à exposição aos resíduos ${ }^{22}$.

\section{CONSIDERAÇÕES FINAIS}

Os resultados desta pesquisa revelaram que os profissionais de enfermagem consideram que há geração de diferentes tipos de resíduos no CC e que, quando identificados e manuseados de forma não adequada, esses materiais podem ocasionar prejuízos para a própria saúde, a de outros profissionais e a dos pacientes.

O manejo dos resíduos gerados no cotidiano laboral desses profissionais é constante. Eles entendem que as etapas de manejo, segregação e acondicionamento são fundamentais para garantir a integridade da saúde do trabalhador e dos pacientes, sendo evidente a preocupação ao realizar o manuseio adequado dos resíduos.

Os discursos dos participantes salientaram que estes se sentem mais expostos aos resíduos biológicos e perfurocortantes durante a execução de suas atividades laborais e reconhecem que essas exposições podem gerar impactos em sua saúde, causando prejuízos.

Outro fato apontado pelos participantes foi a falta de atenção de outros profissionais que compõem a equipe de saúde atuante no CC no que concerne ao descarte inadequado dos resíduos. Isso, como consequência, gera insegurança e preocupação.

Portanto, é recomendável que esses profissionais recebam periodicamente orientações e treinamentos sobre o manejo adequado dos RSS, minimizando riscos de exposição aos resíduos gerados com prejuízos à própria saúde, à saúde do paciente, à de outros profissionais e à do ambiente.

Pode-se considerar como limitação do estudo o fato de não terem sido incluídas outras categorias profissionais que integram a equipe do $\mathrm{CC}$, em função do tempo reduzido para a pesquisa.

\section{REFERÊNCIAS}

1. Lima RMS, Duarte GL, Couceiro TCM, Nascimento MKS, Lima LC. Conhecimento dos anestesiologistas da cidade do Recife com relação aos riscos potenciais no centro cirúrgico: estudo transversal. Rev Bras Anestesiol. 2014;64(2):116-20. http://dx.doi.org/10.1016/j. bjan.2013.06.019

2. Januário GC, Carvalho PCF, Lemos GC, Gir E, Toffano SEM. Acidentes ocupacionais com material potencialmente contaminado envolvendo trabalhadores de enfermagem. Cogitare Enferm. 2017;22(1):1-9. http://dx.doi.org/10.5380/ce.v22i1.48893

3. Carvalho M, Gatti MAN, Conti MHS, Vitta A, Marta SN, Simeão SFAP. Qualidade de vida da equipe de enfermagem do centro cirúrgico. Rev Catarse [Internet]. 2014 [acessado em 30 out. 2019];2(1):71-84. Disponível em: http://revistas.faculdadeunicampo.edu.br/index.php/ RevistaCatarse/article/view/304/136
4. Soares LG, Sarquis LMM, Kirchhof ALC, Cruz EDA. Percepção do risco biológico em trabalhadores de enfermagem. Cogitare Enferm. 2013;18(1):36-42. http://dx.doi.org/10.5380/ce.v18i1.31300

5. Silva LS, Valente GSC. Riscos químicos hospitalares e gerenciamento dos agravos à saúde do trabalhador de enfermagem. Rev Pesq Cuidado Fundamental. 2012;(supl.):21-4. http://dx.doi.org/10.9789/21755361.rpcfo.v0.1662

6. Brasil. Ministério da Saúde. Agência Nacional de Vigilância Sanitária (ANVISA). Resolução da Diretoria Colegiada (RDC) n²22, de 28 de março de 2018. Regulamenta as Boas Práticas de Gerenciamento dos Resíduos de Serviços de Saúde e dá outras providências. Diário Oficial da União [Internet]. 2018 [acessado em 30 out. 2019]. Disponível em: http://portal. anvisa.gov.br/documents/10181/3427425/\%282\%29RDC_222_2018_. pdf/679fc9a2-21ca-450f-a6cd-6abc1cb7bd0b 
7. Nunes TSP, Gutemberg ACB, Armando CB, Pinto FF, Lemos MC, Passos JP. Gerenciamento de Resíduos de Serviços de Saúde: uma revisão de literatura. Rev Pesq Cuidado Fundamental. 2012;(supl.):5760. http://dx.doi.org/10.9789/2175-5361.2012.v0i0.57-60

8. Santana JCB, Almeida LG, Oliveira RL, Melo LC, Costa IMS, Silva CCD, et al. Rotina dos profissionais de enfermagem no trabalho com resíduos em saúde em um hospital público. Rev Enferm UFPE. 2013;7(5):133341. http://doi.org/10.5205/reuol.3960-31424-1-SM.0705201304

9. Lefevre F, Lefevre AMC. Pesquisa de representação social: um enfoque qualiquantitativo: a metodologia do discurso do sujeito coletivo. $2^{\text {a }}$ ed. Brasília: Líber Livro; 2012.

10. Brasil. Conselho Nacional de Saúde. Resolução n 466, de 12 de dezembro de 2012 [Internet]. 2012 [acessado em 5 dez. 2018]. Disponível em: http://conselho.saude.gov.br/resolucoes/2012/Res0466.pdf

11. Stehling MC, Cunha LM, Louredo LM, Camargo CG, Haddad JPA, Silva IJ, et al. Gestão de resíduos com risco biológico e perfurocortantes: conhecimento de estudantes de graduação das áreas Biológicas e da Saúde da Universidade Federal de Minas Gerais. REME. 2013;17(3):594600. http://www.dx.doi.org/10.5935/1415-2762.20130044

12. Oliveira AC, Goncalves JA. Acidente ocupacional por material perfurocortante entre profissionais de saúde de um centro cirúrgico. Rev Esc Enferm USP. 2010;44(2):482-7. https://doi.org/10.1590/ S0080-62342010000200034

13. Quinto-Mosquera Y, Jaramillo-Pérez LM, Cardona-Arias JA. Conocimientos y prácticas de los trabajadores de un hospital sobre el manejo de residuos hospitalarios, Chocó, Colombia, 2012. Medicas UIS [Internet]. 2013 [acessado em 22 abr. 2018];26(1):9-20. Disponível em: http://www.scielo.org.co/pdf/muis/v26n1/v26n1a02.pdf

14. Cardoso ACM, Figueiredo RM. Situações de risco biológico presentes na assistência de enfermagem nas unidades de saúde da família. Rev Latino-Am Enferm. 2010;18(3):73-8.
15. Conselho Regional de Enfermagem (COREN). Norma Regulamentadora (NR) n० 32, de 11 de novembro de 2005. Segurança e Saúde no Trabalho em Serviços de Saúde [Internet]. 2005 [acessado em 8 out. 2019]. Disponivel em: https://portal.coren-sp.gov.br/

16. Rezende LCM, Leite KNS, Santos SR, Monteiro LC, Costa MBS, Santos FX. Acidentes de trabalho e suas repercussões na saúde dos profissionais de enfermagem. Rev Baiana Enferm. 2015;29(4):30717. http://dx.doi.org/10.18471/rbe.v29i4.13559

17. Castro RR, Guimarães OS, Lima VML, Lopes CDF, Chaves ES. Management of healthcare waste in a small hospital. Rev Rede Enferm Nordeste. 2014;15(5):860-8. http://doi.org/10.15253/2175-6783.2014000500016

18. Macedo JI, Ferreira MRMN, Betolini DA, Mendes AA, Takayanagui AMM. Medical waste management in a blood center of Paraná state. Rev Bras Ciências Ambientais [Internet]. 2013 [acessado em 8 out. 2018];27:55-60. Disponível em: http://abes-dn.org.br/publicacoes/ rbciamb/PDFs/27-07_Materia_5_artigos344.pdf

19. Takayanagui AMM. Gerenciamento de resíduos de serviços de saúde. In: Philippi JA, editor. Saneamento, saúde e ambiente: fundamento para o desenvolvimento sustentável. Barueri: Manole; 2005. p. 323-74.

20. Mendes AA, Veiga TB, Ribeiro TML, André SCS, Macedo JI, Penatti JT, et al. Medical waste in mobile prehospital care. Rev Bras Enferm. 2015;68(6):1122-9. http://dx.doi. org/10.1590/0034-7167.2015680618i

21. Pinter MG, Jardim DP. Segregação e diminuição de resíduos sólidos no bloco cirúrgico: uma experiência bem-sucedida. Rev SOBECC. 2014;19(4):226-32. https://doi.org/10.5327/Z1414-4425201400040009

22. Lemos MC. Gerenciamento de resíduos de um hospital público do Rio de Janeiro: um estudo sobre o saber/fazer da enfermagem no centro cirúrgico e central de materiais [dissertação]. Rio de Janeiro: Universidade Federal do Estado do Rio de Janeiro; 2012. 